\section{Kinship Revisited}

\author{
Nicholas Evans ${ }^{1} \cdot$ Stephen Levinson ${ }^{2} \cdot$ Kim Sterelny ${ }^{3}$
}

Accepted: 5 July 2021 / Published online: 23 July 2021

(c) Konrad Lorenz Institute for Evolution and Cognition Research 2021

Kinship is central to social life. It connects the biology of reproduction, the social networks of mutual support (and antagonism), the formation of groups, and the cognitive and linguistic representation of family relationships. The development of these connections has almost certainly been central to the expansion of the social universe of early humans and/or their hominin forebears, for human social life is markedly different from the social lives of the great apes. Setting aside the relatively solitary orangutans, the great apes are social too. However, though they vary in the extent and intensity of intergroup hostility, their social worlds are small. With the exception of adolescents (mostly females) who disperse to adjoining groups, a great ape's social universe is the residential group into which it is born, and likewise, their kinship networks are limited to relations within these residential groups.

That is not true of any human community. Some human residential groups are small, especially those of some forager communities. But they are always connected to other groups, part of a larger social network, and so the social worlds of every member of one of these small communities extends beyond their local group (Layton and O'Hara 2010; Layton et al. 2012). Moreover, kin feel positively towards their kin, displaying "prescriptive amity" as Fortes (2006) put it. Thus, as a default, they have some inclination to help, or at least not hinder, their kin. Add this to the facts that humans

Kim Sterelny

kim.sterelny@anu.edu.au

Nicholas Evans

nicholas.evans@anu.edu.au

Stephen Levinson

Stephen.Levinson@mpi.nl

1 ARC Centre of Excellence for the Dynamics of Language (CoEDL), Australian National University, Canberra, ACT, Australia

2 Max Planck Institute for Psycholinguistics, Nijmegan, The Netherlands

3 School of Philosophy, Australian National University, Canberra, ACT, Australia recognize far more kin as kin than do the great apes, and that human kin recognition (and the supportive emotions that go with that recognition) does not depend on co-residence. The consequence is that human kin recognition was and is one of the mechanisms that make possible the larger social universe of the typical human (Chapais 2008). It is hard to overstate the importance of this larger universe. For it is very likely that there is a fundamental and positive connection between the emergence of networked residential groups and the emergence of the extensive cumulative cultural learning that is fundamental to the demographic expansion of our lineage (Premo and Kuhn 2010; Boyd 2016; Henrich 2016). As kin networks grow, the links between nodes grow exponentially, both requiring expanded cognitive capacities and motivating them, with the likelihood that this has played a role in the growing encephalization of the human lineage (Dunbar 2009).

Great ape kin networks are based on shared experience, most fundamentally the shared history of a mother and her offspring, together with some social referencing, as the young great ape takes its lead from the reactions of its mother to others. As Bernard Chapais emphasizes, for all the cultural variability of human kinship systems, that dyadic relationship is fundamental in human social worlds too (Chapais 2013). But in our lineage it is extended and transformed through the multiplication of important dyad types, each with culturally defined reciprocal rights and duties, and with the role of testimony. For many of us, we know of our more distant kin only through report. As we have just seen, this transition is fundamental in the evolution of a distinctively human social life. But kinship is also an ideal model system for understanding the evolution of human cognition, cultural evolution, and gene-culture coevolution.

Kinship is a central vantage point for exploring these more general themes. In particular: (1) kinship categories, in both biology and culture, are quite precisely defined, and so can be applied (in the biological case) across species and (in the cultural case) across communities. Thus they feed into phylogenetic and comparative projects. We can ask across the primates whether fathers recognize their offspring (and 
vice versa), or whether male coalitions depend on kin connection. Across cultures, we can ask (for example) whether cultures that distinguish lexically between parallel and cross cousins tend to have subsistence economies based on herding, or whether matrilineages correlate with inheritance of land through the female line. As well, (2) it is an important though somewhat unusual system for probing the evolution of language itself. In contrast to most lexical items, ${ }^{1}$ kinship terms can be explicitly defined via terms for just a few basic kinship relations. A brother is a male offspring, other than ego, of ego's two parents. A grandchild is a child of a child of ego. Not only are kin terms capable of explicit definition, those definitions embody the central productive principle of language in being iterative.

Once a term has been built from more basic ones, it too then is available to specify further kin categories. Once "uncle" has been introduced as the brother of ego's father, or of ego's mother, or of the male partner of ego's father's sister or the male partner of ego's mother's sister, "uncle" is available, if wanted, to specify the name of a further kin concept, "suncle": the male offspring of an uncle. Once we see this explicit and recursive structure, it becomes obvious that communities have many choices about the division of kinship space into named categories, and we can and should investigate the factors that influence different divisions. We could easily have instead reserved "uncle" for just the nonaffine uncles, having "muncle" instead for the male partner of ego's father's sister or the male partner of ego's mother's sister. Finally, (3) kinship is an important system for exploring the connections between biology, language, and culture.

The basic biology of reproduction, nurturance, and genetic connection is clearly part of the various human kinship packages, but equally clearly, language and culture play crucial and culturally variable roles. There is, for example, a family of cousin-like concepts, and these genuinely designate some form of real genetic connection between ego and the various forms of cousins. But, as discussed in Passmore et al. (2021), from the perspective of English terminology, some of these lump cousins and siblings, while others split the cousin category into cross and parallel cousins (a parallel cousin is the offspring of father's brother or mother's sister). But there are other relationships conceptualized as kin that are not based on genetic relatedness at all: most obviously, the affine kin. Muncles, as defined above, have no distinctive genetic relationship to ego at all, so some English kin terms (and the affinal terms of most other systems) lump genetic and nongenetic categories. Some human social systems

\footnotetext{
${ }^{1}$ Philosophers have found this out the hard way, through the many failed attempts to come up with necessary and sufficient conditions for ordinary-English terms like "knows," "person," "color," "life" as well as concepts from science like "gene" or "experiment."
}

involve systematic mate-exchange (e.g., the preference for a man to marry a mother's brother's daughter) so fusing consanguineal kin with affines. Given the importance of culturally recognized and validated kin connection in mediating cooperation, and in the specification of social norms, this is a striking phenomenon. The fact that in most human (and indeed primate) societies at least one sex tends to move out of the group provides territorially distributed kin, and these have tended to play an important role in reducing intergroup conflict.

For these reasons, it is no surprise that kinship has played a central role in anthropology and linguistics, though, perhaps for reason of fashion, a less central one in recent decades. We think that the time has come to revisit this role in the light of newer and richer theories of the evolution of human social behavior, especially that work deriving from theories of cultural evolution, and new tools, especially those deriving from comparative methods. In the remainder of this brief introduction to the thematic issue on Evolution of Kinship Systems, we will identify the fruits of this return to anthropology's past.

Joan Silk's paper explores the primate roots of kinship systems, and in particular, the interaction between the various mating systems found in primate life (including the cooperative breeders of the South American lineage) and kin recognition (Silk 2020). Such recognition is evidenced behaviorally by positive discrimination; for example, by choosing to support an agent who is actually kin in conflict with others. Mothers always recognize their offspring, and maternal siblings (and half siblings) recognize one another, if they are close enough in birth order for both to be in their mother's social orbit. So Silk focuses on paternal recognition of kin, especially in species whose social organization involves multi-male, multi-female groups, as with the bonobos and chimps, some South American species, and some of the baboons. In these cases, males may well not be able to recognize their offspring; there is, for example, only fairly thin evidence of male chimps recognizing their children. The result is quite limited kin recognition, for if males do not recognize their children, there is likely to be no recognition of other paternally based kin relations. Paternal half siblings will probably not recognize one another. Without recognition of (real or fictional) paternal links, kinship does not offer the community-wide map of social relations found in traditional human groups.

Where Silk's paper surveys the baseline kin recognition mechanisms established in the primate clade as a whole, and how these play out in the different forms of social and sexual organization in primate societies, Robert Layton and Ronald Planer both probe the earlier evolution of kin recognition and kin-supported social behaviors in hominin evolution. Layton's central concern is the limits of experiential kin recognition (Layton 2020). What forms of kin recognition, and 
in what social contexts, require more than shared affiliative history? He aims to identify the forms of social behavior that require kin recognition and kin affiliation that can$n o t$ be supported just by experiential mechanisms. These forms of social behavior depend on language, or something close to language. Layton's hypothesis is that spatially and temporally extended forms of fission-fusion sociality are stable only if supported by these elaborated forms of kinship. Affiliative emotions would need to be reinforced by explicitly recognized and rehearsed named kin relations. His further hypothesis (based on his reading of the archaeological record, and especially the archaeology of raw material movement) is that Homo heidelbergensis lived in these temporally and spatially extended fission-fusion groups. The bold conclusion is that Homo heidelbergensis had language, or something close to it.

Planer's paper complements that of Layton. Planer too argues that merely experiential kinship would not suffice to build larger social networks, and most especially, networks that connected distinct residential groups (Planer 2020). As noted above, such connections are a distinctive and critical difference between the social lives of anatomically modern humans and great apes, and ethnography certainly suggests that recognized kin relationships across residential groups are essential to maintaining those networks. Bernard Chapais has argued that this larger network required only the evolution of pair-bonding, and with it, paternal experiential recognition of kin. With this expanded network, fathers would recognize their own kin in other groups, as their children (probably daughters) migrated out, and, derivatively, come to recognize their daughters' male partners as kin too, thus building peaceful male-male links between bands (Chapais 2013). As Planer points out, this conflates cause with effect. Residential groups must be peaceful for fathers to have continued contact with their daughters in other groups, and to come to recognize and spend time with their daughters' mates in those other groups. Like Layton, Planer argues that recognizing kin across groups was crucial, but that this recognition was explicit and publicly expressed and reinforced. Planer develops a picture of how explicit kin recognition could evolve from fairly minimal linguistic resources, from a fairly simple protolanguage.

Rob Wilson's paper takes us from earlier hominins to anatomically modern humans and from biological evolution to biocultural evolution (Wilson 2020). The Westermarck Effect has been seen as a flat-footedly biological explanation of incest avoidance, alleged to be a hard-wired response driven by selection against the deleterious effects of inbreeding. The recent literature has rejected this account, in part by a critique of its original evidential support, but much more because this explanation seems to deny the importance of culture and the huge cultural variability of what counts as incest. In this paper, Wilson develops a more nuanced version of the Westermarck Effect, grounding the adaptive arguments phylogenetically, reasserting an evidential base for some biological mechanism, and showing how to incorporate culture into a Westermarck-based explanation of incest avoidance. The main aim of the paper is not to explain incest avoidance in itself, but to use the example to undermine a false dichotomy of cultural versus biological traits.

The contribution by Sam Passmore and colleagues is an ambitious paper that focuses on the cultural evolution of linguistically encoded kinship systems (Passmore et al. 2021). We noted above that even setting aside nongenetic kin, there are an enormous number of ways to categorize the space of kinship relations by lumping and splitting in various ways. The received wisdom of anthropology was that these choices are patterned. If there is lumping at the ego generation (for example, lumping siblings and cousins), then there is lumping at generations above and below that of ego, and likewise for splitting. This led to the development of some classic named patterns of kinship terminological choices: Eskimo, Dravidian, Sudanese, and so on. These patterns were identified and named by eyeballing the data (and hand-noting various complications and exceptions). This paper applies newly developed tools to newly developed databases to test for the reality of these patterns, and the extent to which lumping/ splitting choices at one generation predict lumping/splitting choices at others. While there are patterns, and the space of possible kinship terminologies is unevenly occupied, the traditional conceptualization is not vindicated. The paper ends by discussing a serious problem for the project of developing a credible model of the cultural evolution of kinship systems: the ethnographic practice of reporting a single model system while suppressing information about variation in a community in the use of kin terms. Variation at a given time is the fuel for change over time, so this practice removes information critical for explaining the dynamics of kinship. This paper, and indeed the whole special issue, can be read as a manifesto, calling for a return to a more central role of kinship studies in the social sciences, but with an explicitly comparative focus, and making use of more systematic and quantitative data, with new formal tools.

\section{References}

Boyd R (2016) A different kind of animal: how culture made humans exceptionally adaptable and cooperative. Princeton University Press, Princeton

Chapais B (2008) Primeval kinship. Harvard University Press, Cambridge

Chapais B (2013) Monogamy, strongly bonded groups and the evolution of human social structure. Evol Anthropol 22(2):52-65

Dunbar R (2009) The social brain hypothesis and its implications for social evolution. Ann Hum Biol 36(5):562-572

Fortes M (2006) Kinship and the social order. Routledge, New York 
Henrich J (2016) The secret of our success: how culture is driving human evolution, domesticating our species and making us smarter. Princeton University Press, Princeton

Layton R (2020) Kinship without words. Biol Theory. https://doi.org/ $10.1007 / \mathrm{s} 13752-020-00346-7$

Layton R, O'Hara S (2010) Human social evolution: a comparison of hunter-gatherer and chimpanzee social organization. In: Dunbar R, Gamble C, Gowlett J (eds) Social brain, distributed mind. Oxford University Press, Oxford, pp 83-113

Layton R, O'Hara S, Bilsborough A (2012) Antiquity and social function of multilevel social organization among human hunter-gatherers. Int J Primatol 33(5):1215-1245

Passmore S, Barth W, Quinn K, Greenhill SJ, Evans N, Jordan FM (2021) Kin against kin: internal co-selection and the coherence of kinship typologies. Biol Theory. https://doi.org/10.1007/ s13752-021-00379-6

Planer RJ (2020) Towards an evolutionary account of human kinship systems. Biol Theory. https://doi.org/10.1007/ s13752-019-00339-1
Premo LS, Kuhn SL (2010) Modeling effects of local extinctions on culture change and diversity in the paleolithic. PLoS ONE. https:// doi.org/10.1371/journal.pone.0015582

Silk JB (2020) The phylogenetic roots of human kinship systems. Biol Theory. https://doi.org/10.1007/s13752-020-00349-4

Wilson RA (2020) Rethinking incest avoidance: beyond the disciplinary groove of culture-first views. Biol Theory. https://doi.org/10. 1007/s13752-019-00338-2

Publisher's Note Springer Nature remains neutral with regard to jurisdictional claims in published maps and institutional affiliations. 